\title{
Split Fracture: A Complication of Cerclage Wiring of Acute Patellar Fracture
}

\author{
Cho Yau Lo ${ }^{1}$; Tun Hing Lui ${ }^{1, *} ;$ Yan Kit Sit ${ }^{1}$ \\ ${ }^{1}$ Department of Orthopedics and Traumatology, North District Hospital, Hong Kong, China \\ *Corresponding author: Tun Hing Lui, Department of Orthopedics and Traumatology, North District Hospital, 9 Po Kin Road Sheung Shui, Hong Kong, China. Tel: +852-26838023, Fax: \\ +852-26838028, E-mail: luithderek@yahoo.co.uk
}

Received: May 22, 2014; Accepted: July 26, 2014

\begin{abstract}
Introduction: Iatrogenic patellar fracture is reported as a complication of patella procedures, such as medial patellofemoral ligament reconstruction, reconstruction of the anterior cruciate ligament of the knee using bone-patellar tendon-bone technique, and resurfacing the patella in total knee arthroplasty.

Case Presentation: A 65-year-old lady with right patella fracture was treated with open reduction and cerclage wiring. An iatrogenic split fracture was noted during tension of the cerclage wire and was successfully managed by screw fixation of the split fracture.

Conclusions: Split fracture is a rare complication of circumferential cerclage wiring of patellar fracture. The surgeon should be aware of the contributing factors in order to avoid this complication.
\end{abstract}

Keywords: Patella; Fracture; Cerclage Wire; Split

\section{Introduction}

The patella is the sesamoid bone of the knee joint. Its main biomechanical function is to increase the effective lever arm of the quadriceps (1). Patellar fractures constitute approximately $1 \%$ of all fractures (2). Surgical fixation is employed if the extensor mechanism is disrupted or there is more than $2 \mathrm{~mm}$ step off and $3 \mathrm{~mm}$ of displacement $(1,2)$. The treatment aims to preserve the patella bone, restore the joint surface and extensor mechanism, and provide stable fixation for early mobilization $(2,3)$. Anatomical reduction and fixation with different types of tension-band wiring technique is the most commonly performed procedure (2). Complication rate following the fixation of patellar fracture can be up to $60 \%$ (2). The complications include hardware failure, non-union, intra-articular non-union and development of post-traumatic arthritis, deep infection and sinus tract formation, arthrofibrosis and knee stiffness, quadriceps wasting, symptomatic hardware, migration of broken cerclage wire, stress avulsion of the tibial tuberosity, patella baja, patellar necrosis from blood interference, and peripheral nerve disturbance especially of the branches of the saphenous nerve (3-12). The risk of their occurrence may be augmented by patient-, injury-, and treatment-related factors (5). The current paper reported a case of patellar fracture which was complicated by split fracture as a result of cerclage wiring, which was successfully managed by screw fixation and tension band wiring.

\section{Case Presentation}

A 65-year-old lady slipped and fell with direct contusion of her knee. She had right knee pain and swelling after- wards. Clinically, the extensor mechanism of her right knee was lost. Radiographs showed displaced fracture of her right patella (Figure 1). Open reduction and internal fixation was performed. Intra-operatively, it was complicated by a coronal split fracture during tensioning of the cerclage wire. The cerclage wire was reapplied posteriorly. The split fracture was reduced and fixed with five $2 \mathrm{~mm}$ screws. The fixation was augmented with a figure-of-eight tension band wire (Figure 2). Post-operatively, a hinged knee brace was applied. The knee was immobilized in extension for two weeks and gradual mobilization in the brace for another six weeks. Bone densitometry was checked after the operation. The T score was -1.4. The fracture healed eight weeks after the operation (Figure 3). Upon 24 months of follow-up, the range of motion of her right knee was 0 to 100 degrees of flexion. There was some knee discomfort during flexion by irritation of the wire loop. The patient refused removal of the implants.

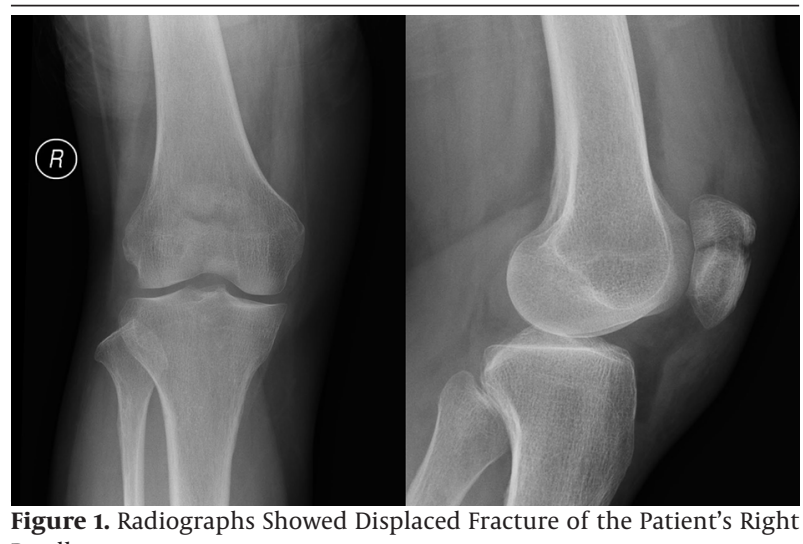
Patella

Copyright (C 2014, Kashan University of Medical Sciences; Published by Kowsar. This is an open-access article distributed under the terms of the Creative Commons Attribution-NonCommercial 4.0 International License (http://creativecommons.org/licenses/by-nc/4.0/) which permits copy and redistribute the material just in noncommercial usages, provided the original work is properly cited. 
Figure 2. The Split Fracture Was Reduced and Fixed With five $2 \mathrm{~mm}$ Screws

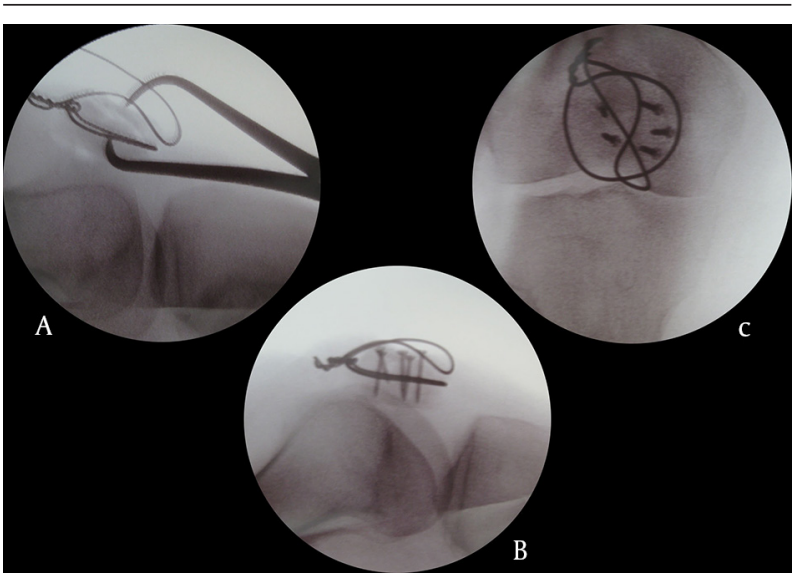

The fixation was augmented with a figure-of-eight tension band wire

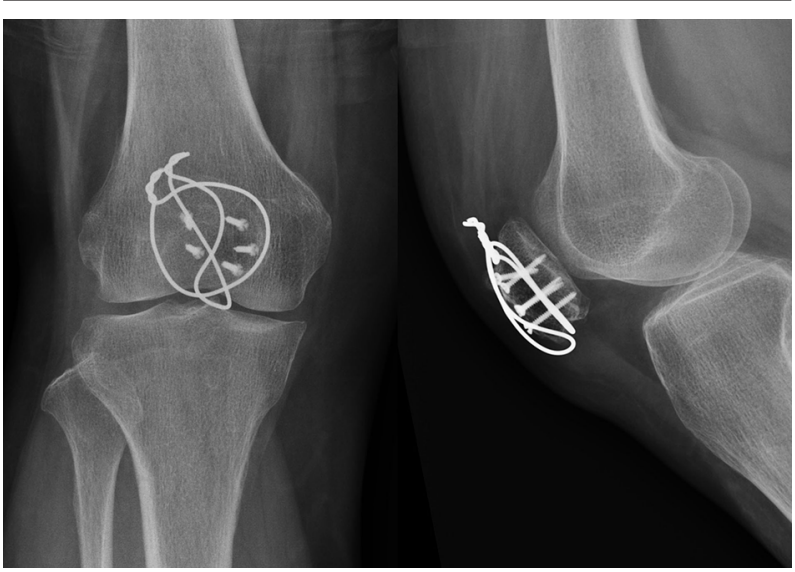

Figure 3. The Radiographs Showed That the Fracture Was Healed

\section{Discussion}

The tension band wiring is the standard operation technique for patellar fracture. It transforms the muscular tensile force into pressure force across the fracture line (13). The main reason for the loss of fixation in tension band wiring is the friction and plastic deformation caused by the wire sliding around the Kirschner wire. As a result, most of the comminuted patellar fractures treated with tension band have to be immobilized for weeks after operation, which would impair the final knee motion (3). On the other hand, Curtis et al. (14) found that the Pyrford technique using a circumferential cerclage wire and an anterior wire looping through the quadriceps tendon modified tension band technique provided a stronger fixation than tension band wiring. It was the reason why circumferential cerclage wire was performed in the current case. However, it was complicated by a coronal split fracture. Iatrogenic patellar fracture is reported as a complication of procedures of the patella, e.g. medial patellofemoral ligament reconstruction (15), reconstruction of the an- terior cruciate ligament of the knee using bone-patellar tendon-bone technique (16), and resurfacing of the patella in total knee arthroplasty (17). The fractures were due to weakening of the patella by the bone tunnel or removal of a part of the bone and the fractures were either longitudinal or transverse in orientation. The fracture pattern and its etiology of the current case were different from the other iatrogenic patella fractures. The cause of split fracture in the current case was believed to be multifactorial including patient factor, implant factor, and technical factor. Firstly, Dual-energy X-ray Absorptiometry (DEXA) scan showed that the patient suffered from osteopenia and the weak bone would predispose iatrogenic fracture during the operation. Secondly, stainless steel wire was used in the current case and it may not conform to the outline of the patella well compared to cable cerclage (3). Point contacts may be present between the wire and the patellar bone. Abnormally high stress to the bone may occur during wire twisting. Moreover, simple wire twisting cannot ensure the application of an exact tension to the fracture in comparison with the cable tensioning device (3), which may result excessive tension of the wire. Thirdly, the circumferential cerclage wire was initially put too anteriorly which was away from the stronger subchondral bone. Moreover, in order to achieve optimum stabilization, the wire was put directly on the bone and the force of the twisting wire was directly transferred to the patellar bone (18). The patella can be cut by the circumferential cerclage wire if the surgeon was not aware of this complication and applied excessive twisting force to the cerclage wire. The iatrogenic split fracture was fixed with screws and tension band wiring. The fracture healed but the patient suffered from anterior knee pain and limitation of the knee flexion. As the patella is in a superficial location, the knot of the stainless steel wire can cause the anterior knee pain $(7,19)$. The limitation of knee flexion was partly due to the initial post-operative immobilization and partly due to the pain caused by the subcutaneous metallic knot, which would hinder rehabilitation (19). This may be solved if suture thread was used instead of metallic wire, since the knot of suture thread would not induce subcutaneous irritation or pain $(1,19-21)$. Although tension-band fixation employing suture thread provides a less rigid construct, satisfactory clinical results and fewer complications are reported $(1,20,21)$. Split fracture is a rare complication of circumferential cerclage wiring of patellar fracture. The surgeon should be aware of the contributing factors in order to avoid this complication.

\section{Authors' Contributions}

Dr. Lo and Dr. Sit prepared the manuscript and performed the literature review; Dr. Lui collected the clinical data and supervised their work.

\section{References}

1. Gosal HS, Singh P, Field RE. Clinical experience of patellar fracture fixation using metal wire or non-absorbable polyester - a study of 
37 cases. Injury. 2001;32(2):129-35.

2. Melvin JS, Mehta S. Patellar fractures in adults. J Am Acad Orthop Surg. 2011;19(4):198-207.

3. Yang L, Yueping O, Wen Y. Management of displaced comminuted patellar fracture with titanium cable cerclage. Knee. 2010;17(4):283-6.

4. Meena S, Nag HL, Kumar S, Barwar N, Mittal S, Singla A. Delayed migration of K-wire into popliteal fossa used for tension band wiring of patellar fracture. Chin JTraumatol.2013;16(3):186-8.

5. Petrie J, Sassoon A, Langford J. Complications of patellar fracture repair: treatment and results. J Knee Surg. 2013;26(5):309-12.

6. Chen YJ, Wu CC, Hsu RW, Shih CH. The intra-articular migration of the broken wire: a rare complication of circumferential wiring in patellar fractures. Changgeng Yi Xue Za Zhi.1994;17(3):276-9.

7. Banks KE, Ambrose CG, Wheeless JS, Tissue CM, Sen M. An alternative patellar fracture fixation: a biomechanical study. J Orthop Trauma. 2013;27(6):345-51.

8. Maeno S, Hashimoto D, Otani T, Masumoto K, Fujita N, Saito S. A Novel Surgical Technique for Patellar Fracture: Application of Extra-articular Arthroscopy With Hanger-Lifting Procedure. Arthrosc Tech. 2013;2(3):e275-9.

9. El-Sayed AM, Ragab RK. Arthroscopic-assisted reduction and stabilization of transverse fractures of the patella. Knee.2009;16(1):54-7.

10. Lazaro LE, Wellman DS, Sauro G, Pardee NC, Berkes MB, Little MT, et al. Outcomes after operative fixation of complete articular patellar fractures: assessment of functional impairment. J Bone Joint Surg Am. 2013;95(14):e96 1-8.

11. Choi HR, Min KD, Choi SW, Lee BI. Migration to the popliteal fossa of broken wires from a fixed patellar fracture. Knee. 2008;15(6):491-3.
12. Hirschmann MT, Wind B, Mauch C, Ickler G, Friederich NF. Stress avulsion of the tibial tuberosity after tension band wiring of a patellar fracture: a case report. Cases J. 2009;2:9357.

13. Labitzke R. [Proper and improper tension band fixation exemplified by patellar fracture]. Chirurg. 1997;68(6):638-42.

14. Curtis MJ. Internal fixation for fractures of the patella. A comparison of two methods. J Bone Joint Surg Br.1990;72(2):280-2.

15. Dhinsa BS, Bhamra JS, James C, Dunnet W, Zahn H. Patella fracture after medial patellofemoral ligament reconstruction using suture anchors. Knee. 2013;20(6):605-8.

16. Milankov M, Rasovic P, Kovacev N, Milovic M, Bojat V. Fracture of the patella after the anterior cruciate ligament reconstruction. Med Pregl. 2012;65(11-12):476-82.

17. Sayeed SA, Naziri Q, Patel YD, Boylan MR, Issa K, Mont MA. Patellar fractures following total knee arthroplasty: a review. J Long Term Eff Med Implants. 2013;23(4):331-6.

18. Baran O, Manisali M, Cecen B. Anatomical and biomechanical evaluation of the tension band technique in patellar fractures. Int Orthop. 2009;33(4):1113-7.

19. Yotsumoto T, Nishikawa U, Ryoke K, Nozaki K, Uchio Y. Tension band fixation for treatment of patellar fracture: novel technique using a braided polyblend sutures and ring pins. Injury. 2009;40(7):713-7.

20. Hughes SC, Stott PM, Hearnden AJ, Ripley LG. A new and effective tension-band braided polyester suture technique for transverse patellar fracture fixation. Injury. 2007;38(2):212-22.

21. Chen $\mathrm{CH}$, Huang HY, Wu T, Lin J. Transosseous suturing of patellar fractures with braided polyester - a prospective cohort with a matched historical control study. Injury. 2013;44(10):1309-13. 